\title{
Reviews
}

\section{Treatment of suprascapular nerve entrapment syndrome}

Joseph D. Leider, BS1, Olivia C. Derise, BS², Kyle A. Bourdreaux, BS², Gregor J. Dierks, BS², Christopher Lee, $\mathrm{MD}^{3}$, Giustino Varrassi, MD, Ph.D., FIPP', William F. Sherman, MD, MBA ${ }^{5}$, Alan D. Kaye, MD, Ph.D ${ }^{6}$

\author{
${ }^{1}$ Georgetown University School of Medicine, Washington DC, ${ }^{2}$ Louisiana State University Health Sciences Center, New Orleans, ${ }^{3}$ Department of \\ Internal Medicine, Creighton University School Of Medicine-Phoenix Regional Campus, Phoenix, AZ, 4 Paolo Procacci Foundation, Via Tacito 7, Rome, \\ Italy, ${ }^{5}$ Department of Orthopaedic Surgery, Tulane Medical Center, New Orleans, LA, ${ }^{6}$ Department of Anesthesiology, Louisiana State University \\ Shreveport, LA \\ Keywords: arthroscopic, nerve entrapment, suprascapular nerve entrapment \\ https://doi.org/10.52965/001c.25554
}

\section{Orthopedic Reviews}

Vol. 13, Issue 1, 2021

\begin{abstract}
Suprascapular nerve entrapment syndrome (SNES) is an often-overlooked etiology of shoulder pain and weakness. Treatment varies depending on the location and etiology of entrapment, which can be described as compressive or traction lesions. In some cases, treating the primary cause of impingement (ie. rotator cuff tear, ganglion cyst, etc.) is sufficient to relieve pressure on the nerve. In other cases where impingement is caused by dynamic microtrauma (as seen in overhead athletes and laborers), treatment is often more conservative. Conservative first-line therapy includes rehabilitation programs, nonsteroidal anti-inflammatory drugs, and lifestyle modification. Physical therapy is targeted at strengthening the rotator cuff muscles, trapezius, levator scapulae, rhomboids, serratus anterior, and deltoid muscle(s). If non-operative treatment fails to relieve suprascapular neuropathy, minimally invasive treatment options exist, such as suprascapular nerve injection, neurostimulation, cryoneurolysis, and pulsed radiofrequency. Multiple treatment modalities are often used synergistically due to variations in shoulder anatomy, physiology, pain response, and pathology as a sole therapeutic option does not seem successful for all cases. Often patients can be treated with non-invasive measures alone; however, injuries refractory to conservative treatment may require either arthroscopic or open surgery, particularly if the patient has an identifiable and reversible cause of nerve compression. Indications for invasive treatment include, but are not limited to, refractory to non-operative treatment, have a space-occupying lesion, or show severe signs and symptoms of muscle atrophy. Open decompression has fallen out of favor due to the advantages inherent in the less invasive arthroscopic approach.
\end{abstract}

\section{INTRODUCTION}

Suprascapular nerve entrapment syndrome (SNES) is an often-overlooked etiology of shoulder pain and weakness. Although rare in the general population, SNES increases significantly in high-risk populations. ${ }^{1-3}$ The suprascapular nerve (SN) originates from the superior trunk of the brachial plexus, where it passes deep to the trapezius toward the scapula to innervate the supraspinatus and infraspinatus muscles. It carries motor nerve fibers to these muscles and sensory nerve fibers from several structures in the posterolateral shoulder. ${ }^{1,4,5}$ The $\mathrm{SN}$ is vulnerable to in- jury at many locations along its path. ${ }^{6}$ The $\mathrm{SN}$ is particularly susceptible to entrapment at the suprascapular notch and the spinoglenoid notch. ${ }^{7}$ Etiologies of SNES can be described as compressive or traction lesions. Traction lesions occur in the setting of ligament tension, hypertrophy, or calcification due to rotator cuff tears, overuse in overhead athletes or overhead laborers. ${ }^{6-8}$ Traction injuries can also be exacerbated or directly caused by anatomical variants of the suprascapular notch, spinoglenoid notch, and accompanying ligaments. ${ }^{9,10}$ Compression injuries occur via mass effect, most commonly from ganglion cysts, soft tissue masses, vascular abnormalities, and malignancies. ${ }^{11}$ They

\footnotetext{
a Corresponding Author

Christopher Lee, MD

St. Joseph Hospital \& Medical Center

Department of Internal Medicine

500 West Thomas Rd

Phoenix, AZ 85013

Chrislee0621@gmail.com
} 
may also result from direct shoulder trauma and iatrogenic complications. $^{1}$

The presentation of SNES is characterized by impairment of sensory and motor innervations to the shoulder.6,10 Those who are symptomatic may present with weakness as well as posterolateral shoulder pain. Symptoms depend largely on the lesion's location, as distal injuries result in isolated symptoms of the infraspinatus muscle. ${ }^{10,12} \mathrm{Muscu}-$ lar atrophy of the supraspinatus and/or infraspinatus tends to differentiate SNES from similarly presenting pathologies. ${ }^{1,7}$

Vague symptomatology and various etiologies make SNES a challenging diagnosis. Once considered a diagnosis of exclusion, SNES can now be confirmed with a thorough history, advanced imaging, and nerve conduction tests. 7,13 MRI is the most valuable imaging study as it allows direct visualization of both the $\mathrm{SN}$ and underlying pathologies such as rotator cuff tears and compressive masses. ${ }^{7,9}$ It also allows the physician to assess supraspinatus and infraspinatus muscle atrophy qualitatively. ${ }^{9}$ Despite the many advantages of MRI, the diagnostic gold standard for SNES remains electromyography (EMG) and nerve conduction velocity (NCV) studies, allowing for localization of the nerve lesion. ${ }^{1,10}$

Treatment of SNES varies depending on the location and etiology of entrapment. In some cases, treating the primary cause of impingement (i.e., rotator cuff tear, ganglion cyst, etc.) is necessary to relieve pressure on the nerve. ${ }^{6,10}$ Injuries refractory to conservative treatment may require either arthroscopic or open surgery. $7,11,13,14$

\section{RISK FACTORS}

\section{OCCUPATIONAL/ATHLETICS}

The relationship between overhead activities and SNES has been well documented in the literature. ${ }^{13}$ Sports such as volleyball, tennis, and swimming increase the risk of dynamic entrapment caused by repetitive compression and subsequent swelling, fibrosis, and demyelination of the SN. ${ }^{13}$ The sling effect describes how certain maneuvers increase stress on the $\mathrm{SN}$ at the spinoglenoid ligament. ${ }^{13,14}$ Protraction and retraction of the scapula during the throwing motion puts significant pressure on the SN. ${ }^{1}$ Cadaveric studies have demonstrated that shoulder adduction and internal rotation increase tension at the inferior portion of the spinoglenoid ligament, directly superior to the SN. ${ }^{3,13}$ This motion, often seen in volleyball spikes and overhead serves, is thought to cause cumulative microtrauma and neuropathy of the SN.1,7,8,11,15 Around 33\% of volleyball players suffer from SNES at some point in their careers. ${ }^{13}$ The greatest tension in the suprascapular ligament is seen during abduction and full rotation of the arm, which is a motion common to many overhead sports. ${ }^{7,12}$ Some researchers have also suggested that SNES can be caused by microemboli formation secondary to repeated compression trauma that damages the vasonervorum. ${ }^{4,13,16}$

\section{PRIMARY SHOULDER INJURIES}

Rotator cuff and labrum injuries are potential causes of SNES. 1,7,13 Massive rotator cuff tears have been shown to increase tension on the SN at the suprascapular notch substantially and spinoglenoid notch as ruptured muscles retract medially. ${ }^{7}$ Paradoxically, some rare events of SNES have also been attributed to rotator cuff repair surgeries. ${ }^{6}$ Cadaveric studies have determined that significant $(1-3 \mathrm{~cm})$ lateral translation of the rotator cuff during repair can increase tension on the SN. $6,13,15$

Following tears of the labrum, synovial fluid can leak into the surrounding tissue forming paralabral ganglion cysts. 1,6 These cysts are formed by a one-way valve mechanism, which allows them to grow in size and compress nearby structures such as the SN. ${ }^{6}$ SNES from ganglion cysts most often occurs at the spinoglenoid notch; however, ganglion cysts can compress the SN at both the spinoglenoid notch or the suprascapular notch depending on the primary labral injury and size of the cyst. 4,11

\section{ANATOMICAL VARIATIONS IN SUPRASCAPULAR NOTCH}

After branching from the superior trunk of the brachial plexus, the SN dives into the shoulder and passes through the suprascapular notch. The scapula forms the osseous portion of the notch. At the same time, the fibrous segment is created by the superior transverse scapular ligament (STSL), which forms the superior border of the foramen. Both the bony notch and the STSL have recognized anatomical variants affecting the incidence of entrapment at the suprascapular notch. ${ }^{11}$

The suprascapular notch can be classified into six anatomical types. ${ }^{1,11}$ These include Type I (wide depression), Type II (wide blunted V shape), Type III (symmetric U shape), Type IV (small V shape), Type V (partial ossified STSL), and Type VI (fully ossified STSL). ${ }^{1,17,18}$ A cadaveric study of 104 scapulae concluded the ossified, Type VI, the suprascapular notch was a risk factor for developing SN impingement. ${ }^{19}$ The study determined that $10-20 \%$ of individuals with a Type VI suprascapular notch had gross impingement. ${ }^{1}$

The shape of the suprascapular notch can be characterized as either U or V-shaped. Based on 616 CT scans of the scapula, Polguj et al. determined the difference between depth and superior transverse diameter of the suprascapular notch was a notable risk factor for developing SNES. ${ }^{11}$ Thus, deep and narrow V-shaped notches increase the risk of SNES. 1,11

As described by Labetowicz et al., there are 3 morphologic variants to the STSL. ${ }^{11}$ These include a fan-shaped ligament (prevalence $=54.6 \%$ ), band-shaped ligament (prevalence $=41.9 \%$ ), and a bifid ligament (prevalence = 3.5\%). 1,7,11,14 of these, the fan-shaped variant was shown to produce the smallest area within the suprascapular notch and is thought to increase the risk of entrapment. ${ }^{12,20}$

\section{VASCULAR ABNORMALITIES}

It is common for the suprascapular vein to accompany the $\mathrm{SN}$ in the suprascapular notch. ${ }^{20}$ Although usually asymptomatic, when the vein becomes congested, the risk for developing SNES increases as the engorged vein limits space for the $\mathrm{SN}$ to pass through the suprascapular notch. ${ }^{1}$

The suprascapular artery has also been documented 
passing through this notch as well. ${ }^{12}$ Additionally, a rare abnormality can also occur where all three structures (suprascapular vein, suprascapular artery, and SN) pass under the STSL. ${ }^{1}$ Crowding of the notch in this situation increases the risk of developing SNES. ${ }^{6}$

\section{PRESENTATION}

The onset of symptoms in SNES typically arises insidiously. ${ }^{6}$ Studies have shown that only $40 \%$ of patients could recall a traumatic event to the shoulder. ${ }^{2,5,13,14}$ Clinical presentation depends on the location and etiology of SN entrapment. 1,6 Dynamic nerve injuries, seen in overhead athletes and laborers, can occur at both the suprascapular notch and the spinoglenoid notch. $1,2,5,15$ Injury at the suprascapular notch can present dull, aching, or burning pain in the posterolateral shoulder that may radiate to the neck or ipsilateral upper extremity. ${ }^{5,14}$ Pain may also be exacerbated with overhead shoulder movements. ${ }^{4}$

Denervation of the supraspinatus and infraspinatus causes weakness in shoulder abduction and external rotation, respectively. $1,12,13$ Weakness may present variably as the teres minor and serratus anterior muscles compensate for infraspinatus muscle weakness. ${ }^{15}$ Due to this compensation, it is critical to inspect the supraspinatus and infraspinatus for signs of atrophy, as it may be the only presenting clinical exam finding. 1,10

Entrapments at the spinoglenoid notch are often caused by dynamic traction injuries or ganglion cysts. However, larger ganglion cysts can entrap the SN at both the suprascapular notch and the spinoglenoid notch. At the spinoglenoid notch, the SN only contains motor nerve fibers to the infraspinatus muscle. ${ }^{10}$ Therefore, entrapments at the spinoglenoid notch cause isolated infraspinatus weakness and atrophy. $7,10,12,15$ The extent of weakness depends on the severity of the nerve entrapment, as well as the degree of compensation from the teres minor. ${ }^{6,12}$ Because sensory nerve fibers join the SN proximal to the spinoglenoid notch. Severe shoulder pain is a far less common presentation in these injuries compared to suprascapular notch entrapment. ${ }^{7}$

\section{DIAGNOSIS}

\section{PHYSICAL EXAM}

Diagnosing SNES includes a history and physical exam followed by shoulder imaging studies. A thorough history and physical exam is critical for assessing possible risk factors while simultaneously ruling out other similar pathologies such as cervical radiculopathy, cervical disk disease, and various rotator cuff injuries. ${ }^{15}$ A physical exam of the shoulder should assess the range of motion, changes in sensation, point tenderness, atrophy, and weakness. When suspecting SNES, the strength of abduction and external rotation should be investigated closely. ${ }^{6}$ Due to the subjective nature of grading muscle weakness, using a handheld dynamometer to assess rotator cuff weakness objectively is prefered. ${ }^{6}$

Physical exams should include an inspection of the shoulder. Supraspinatus atrophy is best appreciated by ex- amining the superior border of the patient shoulders from behind, noting any bilateral asymmetry. ${ }^{7}$ Infraspinatus atrophy can be best assessed while looking down at the scapula with the patient seated closely in front of the physician.

The patient can present with point tenderness at the suprascapular or spinoglenoid notch, depending on the location of the injury. $6,12,13$ Those with lesions in the suprascapular notch may experience tenderness to palpation over the supraspinatus muscles in the supraspinatus fossa, compared to those with spinoglenoid entrapment, which may endorse tenderness deep and posterior to the acromioclavicular joint. 6

Making a diagnosis on a physical exam alone can be challenging because SNES is closely related to injuries of the brachial plexus and rotator cuff. One physical exam maneuver to help diagnose SNES at the suprascapular notch is the suprascapular nerve stretch.6,12 This is performed by having the patient turn their head to face their unaffected shoulder, followed by gentle traction to the affected shoulder. Pain in the posterolateral shoulder is indicative of a positive test. $^{21}$ The empty can test and infraspinatus test may also be positive, but non-specific tests represent many forms of supraspinatus injury. ${ }^{21}$

The physical exam maneuver used to assess spinoglenoid notch entrapment is the cross-arm adduction test. ${ }^{12}$ This test is performed by crossing the patient's extended arm medially across the chest. ${ }^{22}$ A positive test will elicit shoulder pain. ${ }^{22}$ of note, this test will also be positive in acromioclavicular joint arthritis. ${ }^{20}$

\section{IMAGING}

Ultrasound, X-ray, CT, and MRI have strengths and weaknesses in assessing scapular nerve entrapment. Ultrasound is widely considered the first-line imaging study to evaluate peripheral neuropathies because they are clinically efficacious and non-invasive. ${ }^{20,22}$ Furthermore, ultrasound can be used to follow a patient's recovery by assessing muscle texture at regular intervals following treatment. ${ }^{20}$ Ultrasound is a specifically useful modality for evaluating fluidfilled masses such as ganglion cysts and engorged suprascapular veins. ${ }^{23}$ The SN itself may also be directly visualized by ultrasound. ${ }^{22}$ Vascular abnormalities about the suprascapular notch can be visualized using ultrasound with a doppler. ${ }^{8}$ This modality is useful in diagnosing SNES caused by engorged veins or an anatomical defect where the suprascapular artery accompanies the nerve in the suprascapular notch. ${ }^{8}$ In cases where the suprascapular notch is completely ossified, MRI is needed to determine if an artery or vein is accompanying the SN. ${ }^{12}$ MRI is also superior to ultrasound when assessing soft tissue lesions and ganglion cysts. ${ }^{6}$

The usefulness of ultrasound varies widely based on operator experience and lesion depth. ${ }^{23}$ To decrease the depth of the posterior fossa, have the patient put their hand from the affected side on their contralateral shoulder.6,23 Despite the high prevalence of SNES in overhead athletes and the diagnostic utility of ultrasound, there is currently no indication for ultrasound as a screening tool. ${ }^{6}$

Helpful X-ray imaging planes include AP, scapular, lat- 
eral, and stryker. The stryker view is obtained by taking the image with the patient's hand on their head with the X-ray beam angled 10 degrees caudally. ${ }^{9}$ This image helps assess bony abnormalities of the suprascapular notch. ${ }^{9}$ Assessing the anatomical type of the suprascapular notch is particularly important before surgery because the release of an entrapped nerve in a completely ossified (Type VI) suprascapular notch can vary of a fibro-osseous foramen. If an $\mathrm{X}$-ray cannot visualize the suprascapular notch, CT can be used for higher resolution assessment. ${ }^{9}$

MRI is supported in the literature as the ideal imaging modality for analyzing nerves and lesions of the nervous system. ${ }^{12}$ MRI can visualize vascular and bony abnormalities, as well as soft tissue lesions and ganglion cysts. ${ }^{7,8}$ Sotereanos et al. states that MRI has a sensitivity and specificity of $95 \%$ in diagnosing ganglion cysts. ${ }^{7,12}$ Bony structures of the scapula are also well visualized using MRI. Notably, the suprascapular notch and spinoglenoid notch plus their accompanying ligaments, the STSL and spinoglenoid ligament. ${ }^{6,10}$ A cohort of 60 patients with SNES was compared to 47 healthy patients using MRI and determined spinoglenoid notch distension was significantly enlarged in patients with SNES compared to the control group ( $p$ $<0.01)$. They determined that using a spinoglenoid notch distension value of $7 \mathrm{~mm}$ as a cutoff for diagnosing SNES represented a sensitivity of $74 \%$ and a specificity of $78 \% .{ }^{10}$ The same study used MRI to classify fatty atrophy of the supraspinatus and infraspinatus muscles with qualitative Goutallier scores. ${ }^{7,15}$ As a result, Katsuura et al. found a significant proliferation of fatty atrophy in those suffering from SNES vs. control ( $p<0.01)$. These findings further buttress the use of MRI as an imaging modality for assessing muscular atrophy in SNES. $6,7,15$

\section{NERVE CONDUCTION VELOCITY AND ELECTROMYOGRAPHY}

NCV and EMG studies are considered the gold standard for diagnosing SNES. ${ }^{7}$ These tests should be considered when MRI, ultrasound, and physical exam do not yield a diagnosis. ${ }^{6,10}$ Moen et al. concluded NCV and EMG are useful in confirming the diagnosis of suprascapular neuropathy in patients with and without symptoms, physical exam findings, or positive imaging (sensitivity $=74 \%$, specificity $=$ 91\%). ${ }^{15}$ Furthermore, Moen et al. claim NCV and EMG are useful modalities for monitoring a patient's recovery following treatment. ${ }^{15}$ SNES leads to demyelination of the nerve, causing characteristic NCV and EMG findings such as denervation potentials, fibrillations, sharp waves, and prolonged motor latencies. ${ }^{15}$ It is also common to see decreased nerve conduction velocity between Erb's point and the supraspinatus or infraspinatus. ${ }^{15}$ Even with negative NCV, EMG, and imaging, if high clinical suspicion of SNES remains, the diagnosis should not be ruled out.

\section{LOCAL ANESTHETIC}

Local anesthetic injections have shown to be helpful in the diagnosis of SNES and can aid in localizing the point of entrapment. ${ }^{15}$ Although relief of SN pain post-injection can support the diagnosis of SNES at that site. Its diagnostic value is limited by the uncertainty of the precise location and depth of the injection. ${ }^{15}$ No studies have determined the sensitivity and specificity of this test. ${ }^{15}$

\section{ANATOMY}

The suprascapular nerve (SN) is a derivative of the upper trunk of the brachial plexus formed by the ventral rami of C5 and C6 nerve roots that provides both motor and sensory innervation to parts of the upper body. ${ }^{2}$ Beginning in the posterior triangle of the neck, it courses down the dorsal aspect of the scapula through the suprascapular notch and beneath the superior transverse scapular, or suprascapular ligament. It then enters the supraspinous fossa, where it provides motor and sensory innervation to the supraspinatus. After twisting around the lateral spine of the scapula, it finds the infraspinous fossa through an opening called the spinoglenoid notch, thus supplying motor innervation to the infraspinatus muscle. The suprascapular artery and vein run alongside the nerve, but the vessels usually travel above the suprascapular ligament instead. The SN provides somatosensory innervation to the acromioclavicular and glenohumeral joints also. ${ }^{3}$

As the nerve travels, various sites of impingement have been identified. Before the SN enters the notch, it may experience tension from heavy shoulder weight on the supraclavicular area. Soft tissue tumors or scar tissue from clavicular fractures have also caused entrapment before entering the notch. The nerve can also be compressed by anatomic variants of the bony structure of the suprascapular notch, especially since the nerve takes a tortuous route through it. When the SN is in the supraspinous fossa, inflamed tissue from the supraspinatus muscle through excessive use can also cause impingement. Ganglion cysts may protrude in the infraspinous fossa, thereby compressing the SN after innervating the supraspinatus, thereby only causing atrophy of the infraspinatus. The SN courses through numerous bony landmarks and takes a labyrinth of a route to reach its final destination, an impingement in various locations a possible occurrence. ${ }^{4}$

\section{PATHOPHYSIOLOGY}

Although uncommon, suprascapular nerve compression is a possible cause of shoulder numbness, weakness, pain, and dysfunction. Due to its difficult course through bony and small landmarks, many points along its path can serve as sites of compression, especially the suprascapular notch and spinoglenoid notch. It also can experience traction with the transverse scapular ligament. Sports that require a great deal of repetitive overhead arm movement are most commonly involved, such as volleyball, tennis, heavy weightlifting, baseball, and football, to name a few. This trauma can be a direct force or indirectly through damage to the vascular supply to the nerve. Due to variations of the suprascapular notch, transverse scapular ligament, and shape of the scapula itself, some patients may be more susceptible to this type of injury. Iatrogenic injuries, especially after rotator cuff, clavicle, and posterior shoulder repair. ${ }^{5}$ After excluding more common causes of shoulder dysfunction, clin- 
ical findings, and magnetic resonance imaging may lead to suspicion of an SN injury. ${ }^{6}$

The SN contributes to $70 \%$ of the shoulder joint's sensory innervation. ${ }^{7}$ Therefore, SN injury typically presents as weakness of abduction and external rotation of the shoulder and a diffusely dull ache of the posterior and lateral shoulder. One may mistake this for a rotator cuff or spinal issue. Radiation to the lateral arm, neck, or anterior thorax has also been observed. It is noted that injuries at the location of the suprascapular notch tend to be more severe in presentation than the spinoglenoid notch pathologies. Since the nerve has already supplied innervation to the supraspinatus when it reaches the spinoglenoid notch, the only muscle involved is the infraspinatus. Thus, symptoms of poor external rotation of the shoulder may not be present. Injecting a local anesthetic into the suprascapular notch can serve as a diagnostic and a therapeutic test to identify the cause of pain. ${ }^{8}$

\section{CONSERVATIVE MANAGEMENT}

Since many SN neuropathies result from excessive repetitive overhead arm movements, putting those activities to a halt is beneficial to rest the shoulder and nerve and prevent further injury. Without clear evidence of a fracture, spaceoccupying lesion, or immediate need for surgery, non-operative treatment including a rehabilitation program, nonsteroidal anti-inflammatory drugs, and lifestyle modification is first-line therapy. ${ }^{9}$ Physical therapy is targeted at strengthening the rotator cuff muscles, trapezius, levator scapulae, rhomboids, serratus anterior, and deltoid muscle(s). ${ }^{8}$ Scapular movements, such as elevation, pronation, retraction, and depression, are also heavily assessed and fortified. A weak or unstable scapula can cause SN impingement as it passes through the suprascapular notch, so strengthening these muscles can aid in the resolution of symptoms and future damage. ${ }^{8}$

Indications for invasive treatment include, but are not limited to, refractory to non-operative treatment, have a space-occupying lesion, or show severe signs and symptoms of muscle atrophy. Timing is extremely important, as the period between onset of symptoms to when the patient presents can provide a rough estimate of muscle atrophy and denervation. This can be useful when deciding whether it is best to treat nonoperatively or if a more invasive technique is warranted. A rotator cuff tear leading to secondary SN entrapment is common; therefore, treating the problem at hand is more appropriate than an isolated nerve decompression. ${ }^{9}$ It is important to consider the possible long-term effects of choosing a conservative approach rather than immediate decompression. Further nerve damage can cause extensive muscle atrophy and weakness that may not be reversible, so non-operative treatment should be closely monitored to ensure that surgery is not indicated. ${ }^{10}$

\section{MINIMALLY INVASIVE TREATMENTS}

If non-operative treatment fails to provide relief of suprascapular neuropathy, minimally invasive treatment options exist. SN injections can be used to provide an immediate di- agnosis as well as relief. Using anesthetic medications with steroids such as bupivacaine and methylprednisolone acetate can be useful in managing neuropathy. ${ }^{11}$ Most injections are performed at the area of the suprascapular notch, with an ultrasound-guided probe to assist in capturing the nerve instead of the vascular structures nearby. It is noted that locating the superior transverse ligament is extremely useful in identifying the location of the SN. This procedure is typically done with the patient positioned either prone with the arm draped over the side of the table or upright with the ipsilateral hand on the contralateral shoulder. The transducer should be placed parallel to the scapular spine above the suprascapular notch. ${ }^{12}$ This treatment approach can be beneficial in delaying surgery until necessary or appropriate, as well as simply providing short-term or immediate relief of shoulder pain.

If SN blocks do not provide therapeutic relief, another method would include neurostimulation. Neurostimulation is a well-known treatment option for chronic pain from nerve damage in various parts of the body. ${ }^{13}$ Percutaneous neurostimulation is now being studied as a possible modality for treating shoulder pain, specifically since there is not much literature on the topic. There is potential for this technique to provide non-opioid pain relief as a therapeutic option. ${ }^{13}$ The procedure is conducted with the patient lying prone and arms laid in whichever fashion the scapula exhibits a flat surface. Generally, a small incision is made slightly lateral to the spinous process, where an introducer needle is guided to the location of the suprascapular notch. A nerve stimulator device, such as a single quad lead, is then guided through the introducer to the notch, where the $\mathrm{SN}$ is found. Trial stimulation is done during surgery to ensure adequate attachment and correct placement. The rest of the stimulator can be attached to different body areas, such as the thorax and buttocks. The device does not move and the system controls are easily accessible. ${ }^{11}$

Cryoneurolysis is another treatment modality used to provide analgesia until a nerve can "revive." This is achieved through applying exceptionally cold temperatures to the nerve, thus temporarily ablating it and causing Wallerian degeneration. Nitrous oxide or carbon dioxide are highly pressurized gases that can become extremely cold, so they are typically used in this application. Specialized probes are utilized so that no gas enters and remains in the patient's body. Regeneration of the nerve typically takes weeks to months, providing a significant amount of analgesia during this time. Since this can now be done percutaneously, it allows for much broader use of this therapy, which was once only done with complete surgical exposure of the nerve. ${ }^{14}$ Until current advancements, cryotherapy was reserved for treating postoperative pain rather than as its treatment modality. ${ }^{15}$ Using cryotherapy on the SN is an area of focus currently. However, damage to the motor nerves with this technique would lead to muscle weakness and atrophy of the supraspinatus and infraspinatus. Success would be reliant on a selective sensory ablation. More research on this area is necessary.

Pulsed radiofrequency is another method used to control pain caused by suprascapular neuropathy, providing a longacting analgesic effect. It is accomplished by applying short high-voltage bursts of current then allowing heat diffusion 
with longer, silent phases. ${ }^{7}$ This ultimately leads to reversible cell damage, causing nerve fibers to ironically "freeze". ${ }^{16}$ Unlike continuous radiofrequency, which causes extensive damage to neighboring structures, pulsed radiofrequency allows for a finer use with less destruction to the surrounding areas. Since the SN is very small and takes a winding route through the scapula, precisely stimulating the nerve in the most efficacious location poses a great challenge to this therapy. ${ }^{17}$ The addition of ultrasonography has greatly advanced this approach; however, patients may still report pain after the procedure. Endoscopy allows the nerve to be seen clearly, but with that comes a more invasive process. Pulsed radiofrequency has been gaining popularity in treating chronic shoulder pain, especially with the guidance of ultrasonography. Studies are being conducted to evaluate its efficacy, which may show promise for the future. Various combinations and durations of frequencies and currents have been applied to discover the best approach through these experiments.

Chronic shoulder pain has plagued numerous patients throughout the world, and each person is unique in regards to their shoulder anatomy, physiology, pain response, and pathology. Multiple treatment modalities are often used synergistically due to these variations since only one therapeutic option does not seem successful for all cases. Unfortunately, it is common to find patients that have undergone extensive treatment, rehabilitation, and several procedures that proceed to have pain that decreases their quality of life. Although minimally invasive techniques are typically tried before operative treatments, some patients may ultimately need surgery to relieve their shoulder pain.

\section{SURGICAL TECHNIQUES}

Surgery is an important consideration in patients with suprascapular entrapment. Often patients can be treated with non-invasive measures alone. However, surgery becomes the primary treatment modality when those fail, particularly if the patient has an identifiable and reversible nerve compression cause. ${ }^{10}$ In general, there are two modalities to treat SNES - arthroscopic decompression and open decompression. Regardless of modality, the goal of the surgery is to remove any associated compressive lesions or areas. Open decompression has fallen out of favor due to the advantages inherent in the less invasive arthroscopic approach. These advantages include optimal visualization of the $\mathrm{SN}$ and associated structures and do not require transaction of the trapezius at its insertion point. ${ }^{18}$ There is debate whether it is best to correct the surrounding tears or damage in addition to the SSN pathology or if it is best to treat the underlying structural damage to treat the SSN pathology indirectly. ${ }^{1,19-21}$ Ultimately, it is left to the surgeon's discretion and causation of the compression.

\section{ARTHROSCOPIC DECOMPRESSION}

Patients are first placed in either the beach-chair position or lateral decubitus position. Currently, there is no definitive "best" position, and it is up to the surgeon based on their experience and training. ${ }^{22-24}$ Arthroscopic decompression surgery is considered a safe and effective treat- ment modality for suprascapular nerve entrapment. ${ }^{25}$ In one review, researchers found that out of 269 operations, 2 reported complications - superficial infection and adhesive capsulitis. ${ }^{26}$ The few large retrospective analyses of arthroscopic decompression outcomes show that most patients included in the study parameters have a statistically significant improvement in various outcome measures, including a range of motion, shoulder pain, and strength. ${ }^{26-29}$

The following arthroscopic decompression surgical description will be based on the beach-chair position. The patient will be placed supine on the operating table, with the back at 60 to 90 degrees, head secured, and the operative arm resting on a padded Mayo stand or in an arm-positioning device. ${ }^{30}$ Often, traction is placed on the arm to distract the humeral head from the acromion and better visualize the transverse scapular notch. ${ }^{18,31}$

The modern arthroscopic approach utilizes multiple portals - a posterior portal, a lateral subacromial portal, an anterolateral portal, the Neviaser portal, and an SN portal. ${ }^{18}$ The posterior portal is used to achieve diagnostic arthroscopy and intraarticular inspection. ${ }^{29}$ This portal is placed $1 \mathrm{~cm}$ medial and $2 \mathrm{~cm}$ inferior to the posterolateral corner of the acromion. ${ }^{32}$ After initial arthroscopy is complete. The arthroscope is then moved to the lateral subacromial portal. A shaver is typically placed in the anterolateral portal to perform a bursectomy and visualize the coracoacromial ligament better. ${ }^{2,32}$ After identifying the coracoacromial ligament, it is traced to its base to identify the coracoclavicular ligaments, which are then traced to the base of the coracoid process. After identifying the base of the coracoid process, a blunt trocar is placed via the SSN portal, which is located between the spine of the scapula and the clavicle, about $7 \mathrm{~cm}$ medial to the most lateral border of the acromion. ${ }^{32,33}$ The blunt trocar is used to mobilize tissue to visualize the TSL better and ultimately help protect the SSN and suprascapular vessels (SSVs) when transecting the TSL. After the TSL, SSN, and SSVs are well visualized and protected, scissors are advanced through the Neviaser portal and the TSL is transected. ${ }^{18}$ The SSN should be mobilized gently to verify decompression.

\section{OPEN DECOMPRESSION}

There are two approaches to the open decompression procedure, an anterior and a posterior approach. ${ }^{34}$ In general, as with the arthroscopic approaches, the open decompression procedures are well tolerated and have excellent results regarding patient recovery of strength and reduction in pain. ${ }^{34-36}$

The posterior approach requires a prone or lateral decubitus position. ${ }^{36}$ An incision is made $2 \mathrm{~cm}$ superior to the spine of the scapula, and the trapezius fibers are split in a parallel fashion. Using the supraspinatus muscle as a guide, the suprascapular foramen is located via palpation of the superior border of the scapula. Subsequently, the SSV's are identified and protected, and the TSL is transected, typically allowing the SSN to displace out of the suprascapular notch. ${ }^{36}$

If utilizing the anterior approach, the patient will be placed supine on the operating bed facing the contralateral side with the operative arm in slight abduction. ${ }^{35}$ The inci- 
sion is started along with the sternocleidomastoid muscle, about $1 / 3$ of the way up from its insertion, and it is continued inferiorly, parallel with the clavicle. After a distance of about $2 / 3$ of the clavicle has been reached, the incision is extended posteriorly to the spine of the scapula, perpendicular to the clavicle. ${ }^{37}$ The origin of the SSN on the upper trunk of the brachial plexus can be found under the posterior belly of the omohyoid. After identifying the SSN in the cervical triangle, it is dissected along its length to the TSL, at which point the SSVs are protected, and the TSL is transected. ${ }^{37}$

\section{CONFLICT OF INTEREST}

The authors have no conflicts of interest to disclose.

\section{FUNDING STATEMENT}

No funding was received for the completion of this manuscript.

Submitted: June 30, 2021 EST, Accepted: July 03, 2021 EST 


\section{REFERENCES}

1. Fabre T, Piton C, Leclouerec G, Gervais-Delion F, Durandeau A. Entrapment of the suprascapular nerve. J Bone Joint Surg Br. 1999;81-B(3):414-419. doi:10.130 2/0301-620x.81b3.0810414

2. Kostretzis L, Theodoroudis I, Boutsiadis A, Papadakis N, Papadopoulos P. Suprascapular Nerve Pathology: A Review of the Literature. Open Orthop J. 2017;11(Suppl 1):140-153. doi:10.2174/187432500171 1010140

3. Aguirre K, Mudreac A. Anatomy, Shoulder and Upper Limb, Shoulder. StatPearls. Published online 2020:31386649.

4. Al-Redouan A, Holding K, Kachlik D.

"Suprascapular canal": Anatomical and topographical description and its clinical implication in entrapment syndrome. Ann Anat. 2020;(xxxx). doi:10.1016/i.aana $\underline{\mathrm{t} .2020 .151593}$

5. Scully WF, Wilson DJ, Parada SA, Arrington ED. Iatrogenic nerve injuries in shoulder surgery. $\mathrm{J} \mathrm{Am}$ Acad Orthop Surg. 2013;21(12):717-726. doi:10.5435/I AAOS-21-12-717

6. Yoon TN, Grabois M, Guillen M. Suprascapular nerve injury. J Trauma Inj Infect Crit Care. 1982;22(8):720. doi:10.1097/00005373-198208000-00 $\underline{031}$

7. Ergönenç T, Beyaz SG. Effects of ultrasound-guided suprascapular nerve pulsed radiofrequency on chronic shoulder pain. Med Ultrason. 2018;20(4):461-466. doi:10.11152/mu-1543

8. Romeo AA, Rotenberg DD, Bach Jr BR. file://Users/ oliviaderise/Documents/THIR. YNR 7. pd. Suprascapular Neuropathy. Published online 1999:358-367.

9. Moen TC, Babatunde OM, Hsu SH, Ahmad CS, Levine WN. Suprascapular neuropathy: What does the literature show? J Shoulder Elb Surg. 2012;21(6):835-846. doi:10.1016/j.jse.2011.11.033

10. Strauss EJ, Kingery MT, Klein D, Manjunath AK. The Evaluation and Management of Suprascapular Neuropathy. I Am Acad Orthop Surg. 2020;28(15):617-627. doi:10.5435/JAAOS-D-19-00526

11. Kurt E, van Eijk T, Henssen D, Arnts I, Steegers M. Neuromodulation of the suprascapular nerve. Pain Physician. 2016;19(1):E235-E239.
12. Strakowski JA. Ultrasound-Guided Peripheral Nerve Procedures. Phys Med Rehabil Clin N Am. 2016;27(3):687-715. doi:10.1016/j.pmr.2016.04.006

13. Ilfeld BM, Finneran JJ, Gabriel RA, et al. Ultrasound-guided percutaneous peripheral nerve stimulation: Neuromodulation of the suprascapular nerve and brachial plexus for postoperative analgesia following ambulatory rotator cuff repair. A proof-ofconcept study. Reg Anesth Pain Med. 2019;44(3):310-318. doi:10.1136/rapm-2018-100121

14. Gabriel RA, Ilfeld BM. Peripheral nerve blocks for postoperative analgesia: From traditional unencapsulated local anesthetic to liposomes, cryoneurolysis and peripheral nerve stimulation. Best Pract Res Clin Anaesthesiol. 2019;33(3):293-302. doi:1 0.1016/i.bpa.2019.06.002

15. Ilfeld BM, Gabriel RA, Trescot AM. Ultrasoundguided percutaneous cryoneurolysis for treatment of acute pain: Could cryoanalgesia replace continuous peripheral nerve blocks? Br J Anaesth. 2017;119(4):709-712. doi:10.1093/bja/aex142

16. Cristiani F, Hernandez M. Suprascapular Nerve Pulsed Radiofrequency for Chronic Shoulder Pain in a Pediatric Patient. Case Rep Anesthesiol.

2020;2020:1-4. doi:10.1155/2020/5709421

17. Divizia M, Germani G, Urti I, Imani F, Varrassi G, Meloncelli S. Endoscopic neuromodulation of suprascapular nerve in chronic shoulder pain: A case report. Anesthesiol Pain Med. 2020;10(2):0-3. doi:10.5 812/aapm.103624

18. Lafosse L, Tomasi A, Corbett S, Baier G, Willems K, Gobezie R. Arthroscopic Release of Suprascapular Nerve Entrapment at the Suprascapular Notch: Technique and Preliminary Results. Arthrosc - J Arthrosc Relat Surg. 2007;23(1):34-42. doi:10.1016/j.ar thro.2006.10.003

19. Youm T, Matthews PV, El Attrache NS. Treatment of Patients With Spinoglenoid Cysts Associated With Superior Labral Tears Without Cyst Aspiration, Debridement, or Excision. Arthrosc - J Arthrosc Relat Surg. 2006;22(5):548-552. doi:10.1016/i.arthro.2005.1 $\underline{2.060}$

20. Kim SH, Kim SJ, Sung CH, Koh YG, Kim YC, Park YS. Arthroscopic suprascapular nerve decompression at the suprascapular notch. Knee Surgery, Sport Traumatol Arthrosc. 2009;17(12):1504-1507. doi:10.10 07/s00167-009-0858-1 
21. Schroder CP, Skare O, Stiris M, Gjengedal E, Uppheim G, Brox JI. Treatment of Labral Tears with Associated Spinoglenoid Cysts without Cyst Decompression. J Bone Jt Surgery-American Vol. 2008;90(3):523-530. doi:10.2106/JBJS.F.01534

22. Li X, Eichinger JK, Hartshorn T, Zhou H, Matzkin EG, Warner JP. A Comparison of the Lateral Decubitus and Beach-chair Positions for Shoulder Surgery. J Am Acad Orthop Surg. 2015;23(1):18-28. doi:10.5435/JAA OS-23-01-18

23. Peruto CM, Ciccotti MG, Cohen SB. Shoulder Arthroscopy Positioning: Lateral Decubitus Versus Beach Chair. Arthrosc - J Arthrosc Relat Surg. 2009;25(8):891-896. doi:10.1016/i.arthro.2008.10.003

24. Gelber PE, Reina F, Caceres E, Monllau JC. A Comparison of Risk Between the Lateral Decubitus and the Beach-Chair Position When Establishing an Anteroinferior Shoulder Portal: A Cadaveric Study. Arthrosc - J Arthrosc Relat Surg. 2007;23(5):522-528. d oi:10.1016/j.arthro.2006.12.034

25. Millett PJ, Barton RS, Pacheco IH, Gobezie R. Suprascapular nerve entrapment: Technique for arthroscopic release. Tech Shoulder Elb Surg. 2006;7(2):89-94. doi:10.1097/00132589-200606000-0 $\underline{0004}$

26. Momaya AM, Kwapisz A, Choate WS, et al. Clinical outcomes of suprascapular nerve decompression: a systematic review. J Shoulder Elb Surg. 2018;27(1):172-180. doi:10.1016/j.jse.2017.09.0 $\underline{25}$

27. Bhatia S, Chalmers PN, Yanke AB, Romeo AA, Verma NN. Arthroscopic Suprascapular Nerve Decompression: Transarticular and Subacromial Approach. Arthrosc Tech. 2012;1(2):e187. doi:10.1016/ j.eats.2012.07.004

28. Momaya AM, Kwapisz A, Choate WS, et al. Clinical outcomes of suprascapular nerve decompression: a systematic review. J Shoulder Elb Surg. 2018;27(1):172-180. doi:10.1016/i.jse.2017.09.0 $\underline{25}$
29. Davis FB, Katsuura Y, Dorizas JA. A retrospective review of 112 patients undergoing arthroscopic suprascapular nerve decompression. J Orthop. 2020;19:31-35. doi:10.1016/j.jor.2019.11.048

30. Higgins JD, Frank RM, Hamamoto JT, Provencher MT, Romeo AA, Verma NN. Shoulder Arthroscopy in the Beach Chair Position. Arthrosc Tech. 2017;6(4):e1153-e1158. doi:10.1016/j.eats.2017.04.00 $\underline{2}$

31. Gross RM, Fitzgibbons TC. Shoulder arthroscopy: A modified approach. Arthroscopy. 1985;1(3):156-159. doi:10.1016/S0749-8063(85)80002-5

32. Romeo AA, Ghodadra NS, Salata MJ, Provencher MT. Arthroscopic suprascapular nerve decompression: Indications and surgical technique. J Shoulder Elb Surg. 2010;19(2 Suppl):118-123. doi:10.1 016/i.jse.2010.01.006

33. Nord KD, Mauck BM. The New Subclavian Portal and Modified Neviaser Portal for Arthroscopic Rotator Cuff Repair. Arthrosc - J Arthrosc Relat Surg. 2003;19(9):1030-1034. doi:10.1016/i.arthro.2003.09.0 $\underline{35}$

34. Elzinga KE, Curran MWT, Morhart MJ, Chan KM, Olson JL. Open Anterior Release of the Superior Transverse Scapular Ligament for Decompression of the Suprascapular Nerve During Brachial Plexus Surgery. J Hand Surg Am. 2016;41(7):e211-e215. doi:1 $\underline{0.1016 / j . j h s a .2016 .03 .005}$

35. Tender GC, Kline DG. Anterior Supraclavicular Approach to the Brachial Plexus. Oper Neurosurg. 2006;58(Suppl 4):360-365. doi:10.1227/01.NEU.00002 09027.52848.A3

36. Lafosse L, Tomasi A, Corbett S, Baier G, Willems K, Gobezie R. Arthroscopic Release of Suprascapular Nerve Entrapment at the Suprascapular Notch: Technique and Preliminary Results. Arthrosc - J Arthrosc Relat Surg. 2007;23(1):34-42. doi:10.1016/j.ar thro.2006.10.003

37. Shupeck M, Onofrio BM. An anterior approach for decompression of the suprascapular nerve. $J$ Neurosurg. 1990;73(1):53-56. doi:10.3171/jns.1990.7 3.1.0053 